\title{
ERRATA-CORRIGE
}

Avverenza. - Le linee si contano dall'alto della pagina escludendovi la intestatura.

Errori sfuggiti agli Autori nella revisionedellebozze di stampa:

PAgINA

37

239
LINEA

39

I I
IN LUOGO DI :

da un punto qualunque sopra

$$
+\frac{\nabla(x)-[x]}{\log ([x]+1)}
$$

\section{LEGGERE :}

da un punto qualunque del piano tangente in a sopra

$+\frac{\varpi(x)-[x]}{\log ([x]+1)}+\frac{1}{\log 2}$ 